\title{
The Combination of Age, International Standardized Ratio, Albumin and $\gamma$-Glutamyl Transpeptidase (AIAG), Tumor Size and Alpha Fetoprotein (AFP) Stage as the Prognostic Model for Hepatitis B-Related Hepatocellular Carcinoma
}

\author{
Shuangchi Liu' \\ Zhiduan $\mathrm{Xu}^{\prime}$ \\ Zhuling Fang ${ }^{2}$ \\ Dengyong Zhang' \\ Zhongqiang Qin' \\ Longfei Fan' \\ Jiakang Duan (1) \\ Hongxiang Yin' \\ Yigang Zhang' \\ Qing Pang (1D ${ }^{1,3}$ \\ Yi Tan'
}

'Department of Hepatobiliary Surgery, The First Affiliated Hospital of Bengbu Medical College, Bengbu, 233000, Anhui, People's Republic of China; ${ }^{2}$ Department of Medical Laboratory, Bengbu Medical College, Bengbu, Anhui, 233000, People's Republic of China; ${ }^{3}$ Department of Hepatobiliary Surgery, The First Affiliated Hospital of Xi'an Jiaotong University, Xi'an, 7|006I, Shaanxi, People's Republic of China
Correspondence: Yi Tan; Qing Pang Department of Hepatobiliary Surgery, The First Affiliated Hospital of Bengbu Medical College, Bengbu, Anhui, 233000, People's Republic of China Email doctortanyi2007@I26.com; portxiu2@I26.com
Background: Advanced liver fibrosis can lead to cirrhosis, portal hypertension and liver failure. Besides, advanced liver fibrosis and cirrhosis are the major risk factors for hepatocellular carcinoma (HCC). Almost all patients with HCC also have liver cirrhosis. This study aims to predict the survival rate of hepatitis B-related hepatocellular carcinoma (HCC) by age, international standardized ratio, albumin and $\gamma$-glutamyl transpeptidase (AIAG), an indicator measuring the degree of cirrhosis.

Methods: A total of 501 hepatitis B-related HCC patients experiencing radical surgery were analyzed, retrospectively. General data about demographics and labs were collected at the date of diagnosis to calculate AIAG [age, international standardized ratio (INR), albumin and gamma-glutamyl transferase (GGT)]. The Kaplan-Meier curves and Cox analysis were used to evaluate overall survival (OS) and recurrence-free survival (RFS). The C-index was calculated in R software (version 4.0.3) to evaluate the accuracy of the prognostic model.

Results: During a median follow-up period of 30 months, $31.1 \%(156 / 501)$ of the patients died, and $34.3 \%(172 / 501)$ experienced the recurrence of HCC. Compared with patients with lower AIAG score, patients with higher AIAG score had higher Child-Pugh grade and were at higher Barcelona Clinic Liver Cancer (BCLC) stage (both $P<0.05$ ). Multivariate analysis suggested that GGT, alpha fetoprotein (AFP), tumor size, BCLC stage and AIAG grade were independent predictors of OS and RFS. Furthermore, the combined use of tumor size, AFP and AIAG stage could predict survival significantly better (C-index $=0.710,95 \%$ CI: 0.669 $0.751)$ than BCLC stage.

Conclusion: AIAG is significantly associated with survival of HCC patients, and provides additional prognostic information for patients with HCC. Our findings suggest that the combination of AIAG, tumor size and AFP stage has a better predictive value for the prognosis of patients with hepatitis B-related hepatocellular carcinoma. However, it is necessary for more external evidences to determine clinical utility.

Keywords: AIAG, hepatocellular carcinoma, survival, prognosis

\section{Introduction}

Hepatocellular carcinoma (HCC), the most common liver malignant tumour, is the world's third leading cause of death of men and the fifth leading cause of death of women. ${ }^{1,2}$ Although the long-term rapid increases of HCC mortality have slowed down 
in women and stabilized in men, the HCC prognosis is still usually poor due to the bad effectiveness of the existing treatments. The reported 5-year overall survival of HCC is approximately $5-6 \%{ }^{1,2}$ The Hepatitis $\mathrm{B}$ and $\mathrm{C}$ virus (HBV and $\mathrm{HCV}$ ) are the main risk factors of $\mathrm{HCC}$, especially in Asia. ${ }^{3,4}$ However, in some regions with a low prevalence of $\mathrm{HBV}$ and $\mathrm{HCV}$, alcohol and non-alcoholic fatty liver disease (NAFLD) are the most usual risk factors of HCC.,

Advanced liver fibrosis results in cirrhosis, portal hypertension, and liver failure. In addition, advanced liver fibrosis and cirrhosis are the major risk factors for HCC. ${ }^{7}$ Ninety percent of HCC patients suffer cirrhosis, and one-third of cirrhotic patients finally develop into HCC in their lifetime. ${ }^{8,9}$ Besides, the risk of HCC recurrence after surgery obviously increases when patients have cirrhosis. ${ }^{10}$ Considering that cirrhosis is closely related to the oncogenesis and the outcome of HCC, hepatologists are trying to develop some effective models predicting the status of liver cirrhosis and the prognosis of liver cancer.

Child-Pugh (CP) score, Modified Child-Pugh (MCP) score, Albumin-bilirubin (ALBI) grade, Barcelona Clinic Liver Cancer (BCLC) staging, etc. have been developed to predict survival of HCC. ${ }^{11,12}$ However, their predictive accuracy was not very ideal. ${ }^{11,12}$ In addition, several noninvasive models with high diagnostic potential can be regarded as valuable predictive tools for cirrhosis in patients with HBV or HCV, which is confirmed by numerous data. ${ }^{13,14}$ In recent years, some studies also have reported that some cirrhosis-associated noninvasive models are valuable to predict the survival rate of HCC. ${ }^{14,15}$ Age, international standardized ratio (INR), albumin (ALB) and $\gamma$ - Glamyltransspeptidase (GGT) (AIAG) have been verified as an accurate indicator of liver cirrhosis for patients with HBV infection. ${ }^{16}$ Some studies suggest that the abnormal expression of some genes in the cirrhosis tissue can promote the formation of $\mathrm{HCC}$, and almost all patients with hepatocellular carcinoma (HCC) also have liver cirrhosis. ${ }^{17}$ However, so far, the predictive ability of AIAG in the survival rate of HCC patients has never been investigated. Therefore, in our study, a total of 501 hepatitis B-related HCC cases were analyzed to primarily explore the roles of AIAG in predicting survival rate of $\mathrm{HCC}$ patients.

\section{Methods}

This study was conducted in accordance with the declaration of Helsinki. This study was conducted with approval from the Ethics Committee of Bengbu Medical College and Xi'an Jiaotong University. Written informed consent was obtained from the participants.

\section{Patients}

A total of 501 hepatitis B-related HCC subjects receiving the radical treatment in the Department of Hepatobiliary Surgery from January 2009 to December 2018 were analyzed, retrospectively. Among them, 355 subjects were from the First Affiliated Hospital of Xi'an Jiaotong University, and 146 subjects were from the First Affiliated Hospital of Bengbu Medical College.

\section{Inclusion and Exclusion Criteria}

The inclusion criteria were as follows: 1) patients were diagnosed as HCC by histological pathology; 2) patients first received radical hepatectomy, which is the removal of the entire tumor, along with the regional tissue and lymph nodes; 3) hepatitis B surface antigen was positive.

The exclusion criteria were as follows: 1) individuals with the lack of complete clinical data or follow-up information; 2) Patients with non-radical therapy, receiving surgery for $\mathrm{HCC}$ previously, with $\mathrm{HCV}$ (coinfection), with concomitant cholangiocarcinoma, or with extrahepatic metastasis.

\section{Data Collection}

The following information from the patients' electronic medical records was collected: age, sex, ascites, hepatic encephalopathy, preoperative laboratory data [alanine aminotransferase (ALT), serum total bilirubin (TBIL), aspartate aminotransferase (AST), GGT, platelet count (PLT), INR, ALB, and alpha fetoprotein (AFP)], tumor characteristics (maximum diameter and number) and postoperative pathological outcomes. The data before surgery were collected to calculate AIAG according to the following formula:

$$
\begin{aligned}
P= & -7+0.03 \times \text { age }+9 \times I N R-0.08 \times A L B \\
& +0.004 \times G G T
\end{aligned}
$$

$A I A G=e^{P} /\left(1+e^{P}\right)^{14}$

\section{The Diagnosis and Follow-Up of HCC}

HCC was initially evaluated by medical history, AFP, and radiographic results including $\mathrm{CT}, \mathrm{MRI}$, and/or ultrasound, and was confirmed by postoperative pathology. The patients were followed up every 2 months for the 1 st year, every 4 months for the 2 nd year, and every 6 months thereafter. Patients with the recurrence of HCC after surgery received hepatic resection again, 
radiofrequency ablation (RFA) or transcatheter arterial chemoembolization (TACE).

\section{Statistical Analysis}

Predictive Analytics Software (PASW), version 23.0 software (SPSS Inc., Chicago, Illinois, USA), was used to do the analysis. The $\mathrm{C}$-index was calculated in $\mathrm{R}$ software (version 4.0.3). The continuous variables with skewed distribution were expressed as median (range), and the variables with normal distribution were expressed as mean value \pm SD (Kolmogorov-Smirnov test, $P>0.05$ ). For the missing values, the values of adjacent points of the linear trend were used to represent them, and to do the analysis and calculation. Comparisons between groups were performed by the $t$-test, Wilcoxon, or $\chi^{2}$ test, as appropriate. The overall survival (OS) and recurrencefree survival (RFS) were observed primarily, and they were initially evaluated by the Log rank test and the Kaplan-Meier curves. The multivariate Cox regression model was applied to all variables with statistical significance in the univariate survival analysis to select the remaining significant factors. The area under the receiver operating characteristic curve (AUC) and concordance index (C-index) were calculated to test the discriminatory powers of for predicting survival. When the larger the $\mathrm{C}$-index was, the higher the accuracy of prognostic prediction was. In general, the value of $\mathrm{C}$-index between 0.50 and 0.70 meant a low accuracy, between 0.70 and 0.90 meant a medium accuracy, and higher than 0.90 meant a high accuracy. $P$ value less than 0.05 was considered as statistical significance.

\section{Results}

\section{Patients' Characteristics}

Among the 501 enrolled patients, there were 394 men and 107 women, with the mean age of $53.4 \pm 10.9$ years. On admission, 457 patients had Child-Pugh grade A and the others had grade B. During a median follow-up periods of 30 months, $31.1 \%(156 / 501)$ of the patients died and $34.3 \%(172 / 501)$ experienced a relapse. The inclusion process of eligible patients is showed in Figure 1. Finally, 501 patients were enrolled. The demographic characteristics of the patients are showed in Table 1.

\section{Determining the Cut-off Value of AIAG}

When the difference between sensitivity and 1-specificity was maximum, the cut-off value was optimal. The ROC curve of AIAG indicated that 0.91 was the optimal cut-off value, with $37.2 \%$ sensitivity and $75.7 \%$ specificity (Supplementary Table 1). AIAG, as a significant indicator, was used to predict HCC mortality, with 0.574 of the area under the curve value (95\% CI: $0.521-0.628, p=0.008)$.

\section{Associations Between the AIAG and Clinicopathologic Features}

Demographic data, serologic tests, tumor characteristic indicators and tumor stages stratified by AIAG are summarized in Table 1. Child-Pugh grade and BCLC stage of cases with AIAG $>0.91$ was obviously different from cases with $A I A G \leq 0.91$. Cases with different AIAG scores had obviously different levels of ALT, AST and PLT.

\section{Prognosis of the Entire Cohort}

The Kaplan-Meier cumulative OS and RFS curves are shown in Figure 2A and B. The median survival time was 65 months for all cases, and the 1-year, 3-year, and 5-year OS of patients were $83.0 \%, 65.1 \%$ and $54.8 \%$, respectively. The Kaplan-Meier cumulative OS and RFS curves of patients stratified by AIAG score are shown in Figure $3 \mathrm{~A}$ and $\mathrm{B}$, the 1-year, 3-year, and 5-year OS of patients with high AIAG grade were $75.7 \%, 49.7 \%$ and $43.5 \%$, respectively, and the 1-year, 3-year, and 5-year OS of patients with low AIAG grade were $85.5 \%, 70.6 \%$ and $58.9 \%$, respectively. Log rank test showed that there was a significant difference in survival among different AIAG groups in HCC.

\section{Survival Predictors}

The ROC curve analysis revealed that the OS and RFS were significantly different in patients with different BCLC stage (Figure 4A and B, both $p<0.05$ ). Univariate Cox regression analysis was conducted on the 13 variables, and the results are shown in Table 2. Univariate analysis revealed that ALT, AST, TBIL, PLT, AFP, tumor size, tumor number, BCLC stage, Child-Pugh grade, and AIAG grade were significantly correlated to OS and RFS (Table 2).

Then, multivariate Cox regression analysis was conducted on the above 10 variables. Enter method was used to screen independent variables, and correlation of regression coefficients was calculated. Multivariate analysis suggested that AIAG, tumor size and AFP were independent impact factors in predicting OS and RFS (Table 3). 


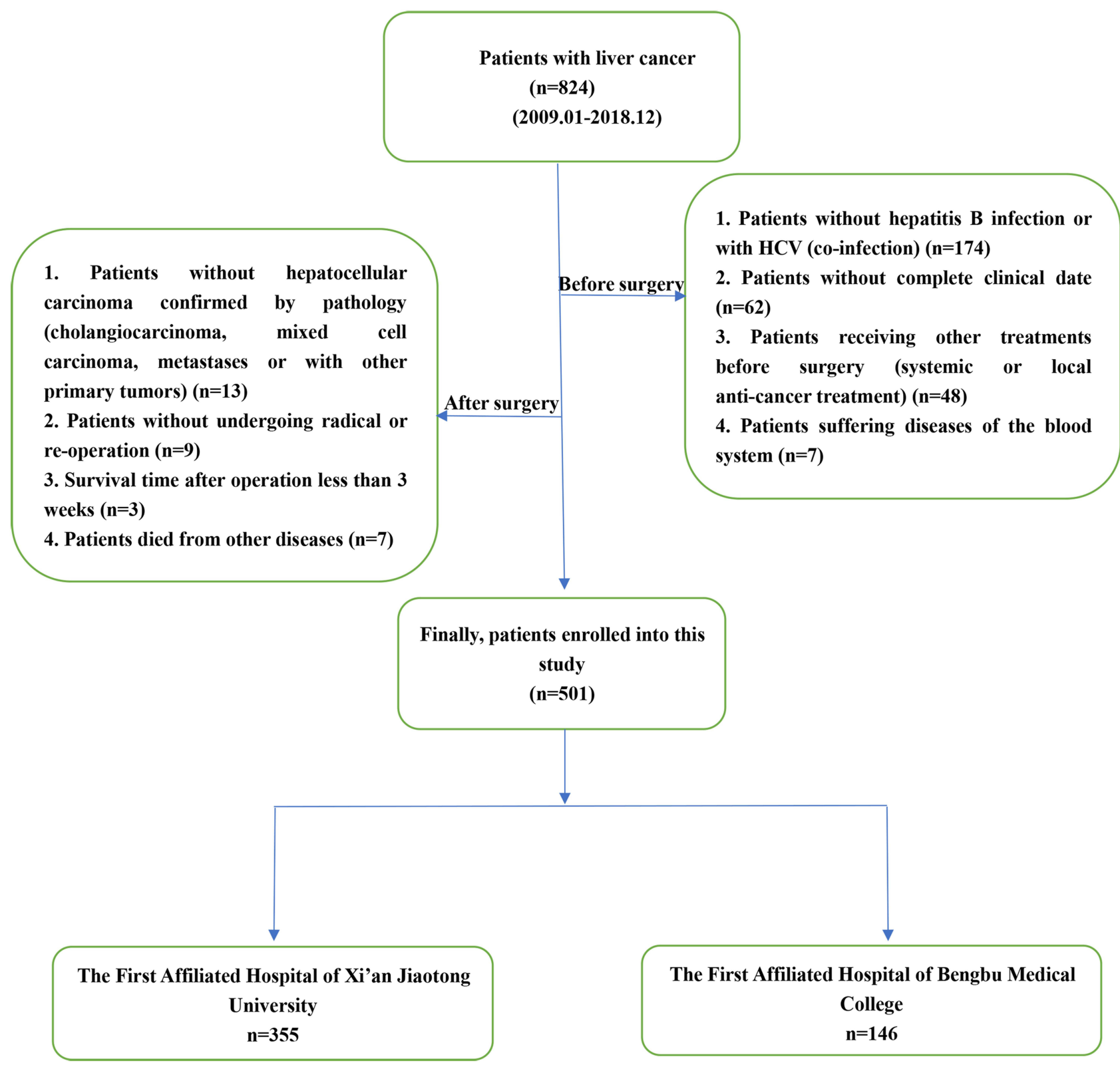

Figure I Flow chart showing the inclusion process of eligible patients in the study.

Comparing the Accuracy of Predicting Survival Between the AIAG and the

\section{Classic BCLC Stage}

In this present study, the value of the C-index of AIAG was lower than that of BCLC stage (Table 4). A large number of studies showed that tumor size and AFP were crucial prognostic factors of HCC. ${ }^{18-20}$ The similar result was also observed in this present study. When combined with tumor size and AFP, the AIAG $(0.705,95 \%$ CI: 0.655-0.755) indicated a higher rank than BCLC staging system (Table 4).

\section{Combination of AIAG, Tumor Size and AFP} Logistic regression was performed, and the ROC curves were drawn as shown in Figure 5. The area under the curve (AUC) of each subgroup is shown in Table 4. The AUC of AIAG+ tumor size+ AFP stage was $0.728(95 \%$ CI: 0.676-0.769), which was higher than that of BCLC stage of 0.654 (95\% CI: 0.602-0.705).

Cox regression is performed, and the $\mathrm{C}$ index is shown in Table 5. The combination of AIAG, tumor size and AFP presented a medium accuracy (AUC $>0.70$ ) in predicting survival rate of HCC. 
Table I Basic Characteristics of Patients with Hepatitis B-Related Hepatocellular Carcinoma Stratified According to Level of the AIAG

\begin{tabular}{|c|c|c|c|c|}
\hline \multirow[t]{2}{*}{ Variables } & \multirow[t]{2}{*}{ Overall } & \multicolumn{2}{|c|}{ AIAG } & \multirow[t]{2}{*}{$P$} \\
\hline & & $\leq 0.91$ & $>0.91$ & \\
\hline \multicolumn{5}{|l|}{ Sex } \\
\hline Male & 394 & 282 & 112 & 0.937 \\
\hline Female & 107 & 77 & 30 & \\
\hline Age (years) & $53.4 \pm 10.9$ & $52.7 \pm 11.3$ & $55.3 \pm 9.6$ & 0.015 \\
\hline ALT (U/L) & $35(7-13 \mid 5)$ & $30(7-1315)$ & $53(13-1068)$ & $<0.001$ \\
\hline AST (U/L) & $38(I I-1247)$ & $33(11-1075)$ & $54(12-1247)$ & $<0.001$ \\
\hline GGT (U/L) & $60(3-1579)$ & $52(3-538)$ & $89(12-1579)$ & $<0.001$ \\
\hline TBIL (mol/L) & $\mid 4(2-43 \mid 2)$ & $13(2-107)$ & $18(3-43 \mid 2)$ & $<0.001$ \\
\hline ALB (g/L) & $40(19-72)$ & $4 I(19-72)$ & $34(19-51)$ & $<0.001$ \\
\hline INR & I.I(0.7-27.9) & $1.0(0.7-1.2)$ & I.2((1.0-27.9) & $<0.001$ \\
\hline PLT $\left(\times 10^{9} / \mathrm{l}\right)$ & $|4|(3 \mid-575)$ & $\mid 46(3 \mid-438)$ & $117(37-575)$ & $<0.001$ \\
\hline \multicolumn{5}{|l|}{ AFP } \\
\hline$>200 \mathrm{ng} / \mathrm{MI}$ & 196 & 140 & 56 & 0.930 \\
\hline$\leq 200 \mathrm{ng} / \mathrm{mL}$ & 266 & 189 & 77 & \\
\hline \multicolumn{5}{|l|}{ Child-Pugh } \\
\hline A & 457 & 349 & 108 & $<0.001$ \\
\hline B & 44 & 10 & 34 & \\
\hline \multicolumn{5}{|l|}{ Ascites } \\
\hline Yes & 48 & 31 & 17 & 0.387 \\
\hline No & 412 & 291 & $|2|$ & \\
\hline \multicolumn{5}{|l|}{ Tumor size } \\
\hline$>5 \mathrm{~cm}$ & 289 & 207 & 82 & 0.986 \\
\hline$\leq 5 \mathrm{~cm}$ & 212 & 152 & 60 & \\
\hline \multicolumn{5}{|l|}{ Tumor number } \\
\hline Multiple & 65 & 46 & 19 & 0.856 \\
\hline Single & 430 & 309 & 21 & \\
\hline \multicolumn{5}{|l|}{ Cirrhosis } \\
\hline Yes & 291 & 200 & 91 & $<0.001$ \\
\hline No & 210 & 160 & 50 & \\
\hline \multicolumn{5}{|l|}{$\mathrm{BCLC}$} \\
\hline 0 & 359 & 183 & 57 & 0.029 \\
\hline$A / B$ & 142 & 176 & 85 & \\
\hline
\end{tabular}

Abbreviations: AIAG, age, international standardized ratio, albumin, $\gamma$-glutamyl transpeptidase; ALT, alanine aminotransferase; AST, aspartate aminotransferase; GGT, $\gamma$ glutamyl transpeptidase; TBIL, total bilirubin; ALB, albumin; INR, international standardized ratio; PLT, platelet; AFP, $\alpha$-fetoprotein; BCLC, Barcelona Clinic Liver Cancer.

\section{Discussion}

With more and more people suffering various hepatitis, especially hepatitis B or C, excessive alcohol, and obesity worldwide, HCC cases gradually increase. This kind of malignant tumor has grown into being one of the most common diseases threatening people's lives. Cirrhosis, as the intermediate disease, connects risk factors with HCC. Therefore, we are struggling to develop a cirrhosis-related noninvasive model to predict the HCC's prognosis.

In the past, Child-Pugh (CP) score, Modified ChildPugh (MCP) score, Albumin-bilirubin (ALBI) grade, Barcelona Clinic Liver Cancer (BCLC) staging, etc. were 

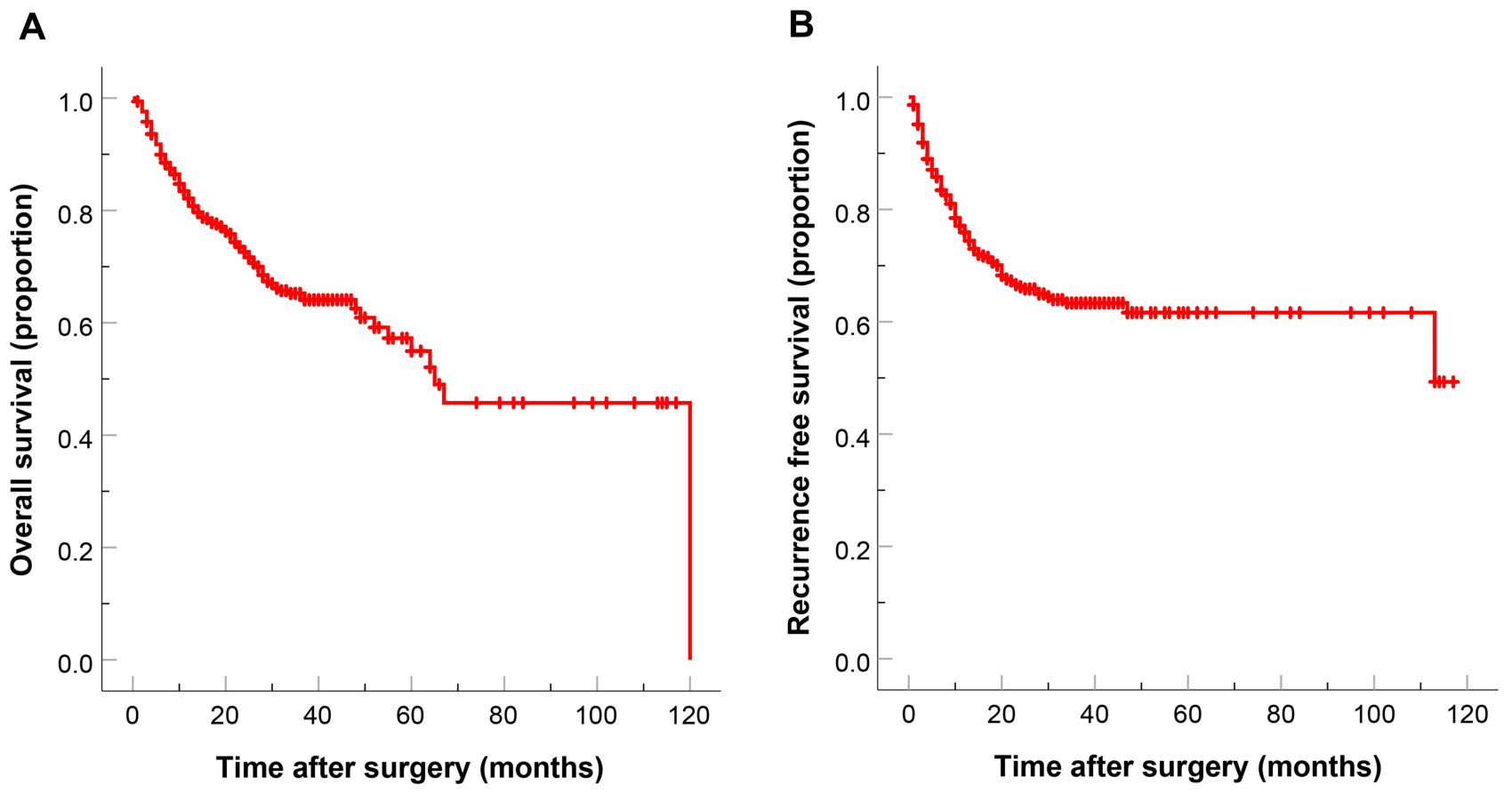

Figure 2 Kaplan-Meier cumulative overall survival (A) and recurrence-free survival (B) curves of the study population.
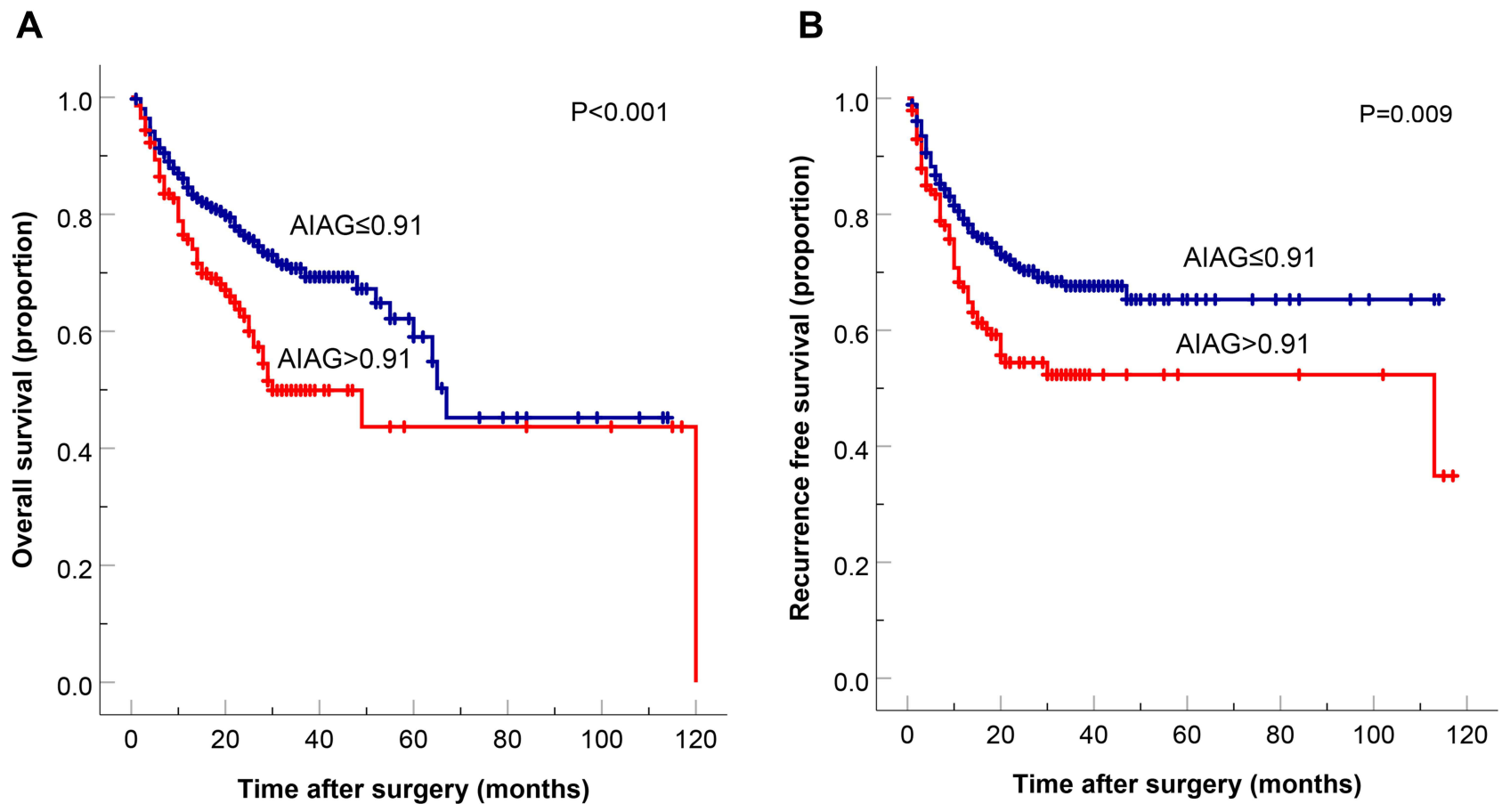

Figure 3 Kaplan-Meier cumulative overall survival (A) and recurrence-free survival (B) curves of patients stratified according to the AIAG.

developed to predict survival of HCC. ${ }^{11,12}$ However, their predictive accuracy was not very ideal. ${ }^{11,12}$ Therefore, it is necessary to explore the new methods to do the prediction.

AIAG is a cirrhosis-associated noninvasive indicator, and past researches showed the accuracy of AIAG in diagnosing cirrhosis combined with chronic hepatitis B. ${ }^{16}$ In our study, hepatitis B-related HCC cases were exclusively enrolled, and the prognostic significance of the AIAG was assessed. It was found that the AIAG was independently associated with the consequences of HCC 

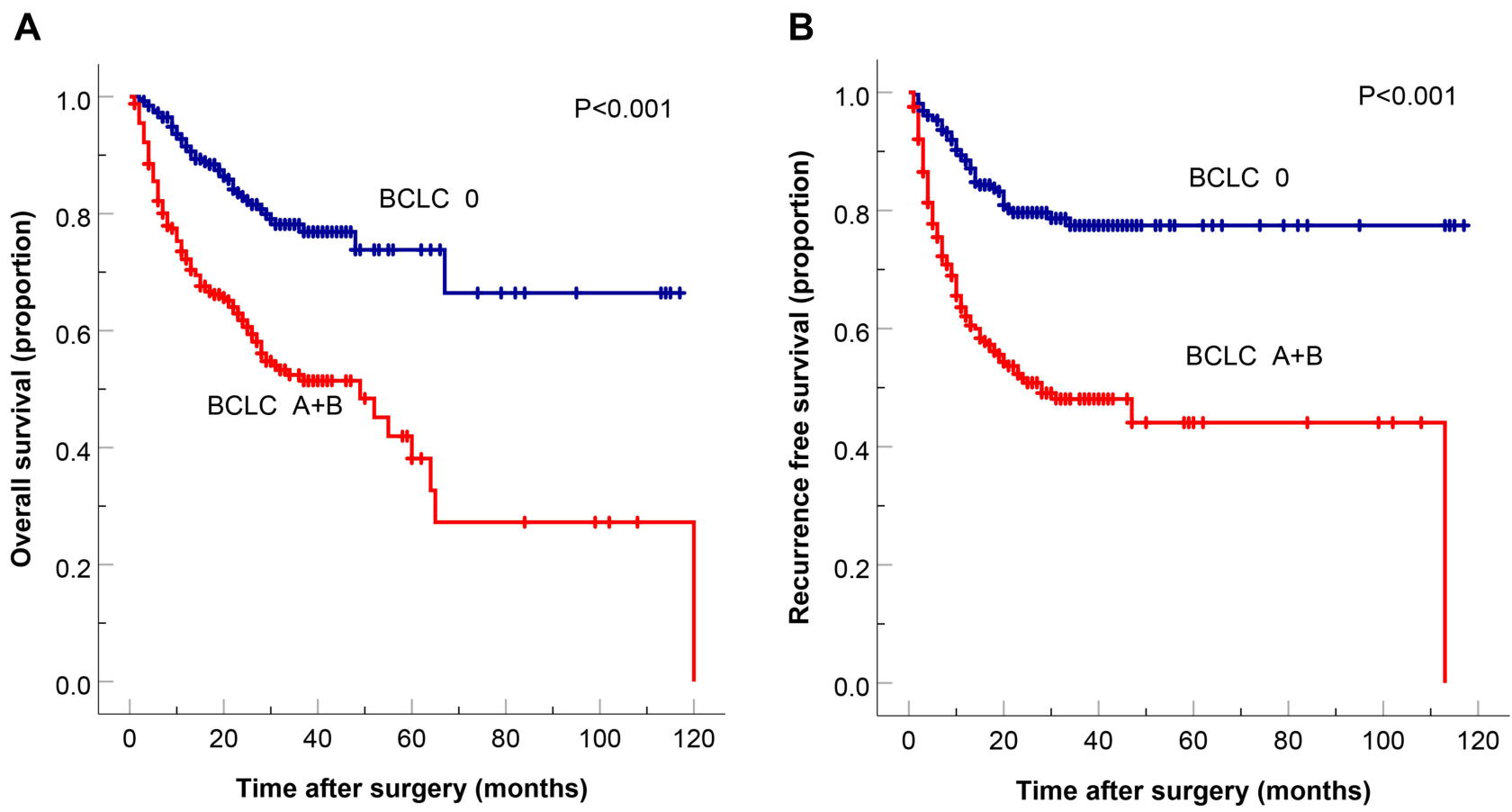

Figure 4 Kaplan-Meier cumulative overall survival (A) and recurrence-free survival $(\mathbf{B})$ curves of patients stratified according to the BCLC stage.

when this binary variable of the noninvasive indicator was adopted. Patients with a high level of AIAG also had high value of ALT, AST, TBI, Child-Pugh grade and BCLC stage, which were independent risk factors for OS in the univariate analysis. Patients with the AIAG value greater than 0.91 had higher probability of experiencing cirrhosis than patients with the AIAG value less than $0.91(64.5 \%$ vs $55.6 \%, P<0.001)$. Although several studies indicated that cirrhosis conditions were related to the OS of HCC, there was no significant relation between them in our study, which might be due to the small sample size of our study. $^{21,22}$ Studies on HCC suggested that cirrhosis, as a serious clinical problem, was a risk factor for RFS, which was further confirmed by our study. Some factors,

Table 2 Univariate Analysis of Factors Associated with Overall Survival and Recurrence-Free Survival of Patients with Hepatitis B-Related Hepatocellular Carcinoma

\begin{tabular}{|c|c|c|c|c|}
\hline \multirow[t]{2}{*}{ Variables } & \multicolumn{2}{|c|}{ os } & \multicolumn{2}{|c|}{ RFS } \\
\hline & HR (95\% Cl) & $P$ & HR (95\% Cl) & $P$ \\
\hline Sex (male/female) & $1.024(0.697-1.504)$ & 0.905 & $1.035(0.705-1.520)$ & 0.861 \\
\hline ALT $(U / L)$ & $1.001(1.000-1.002)$ & 0.038 & $1.001(1.000-1.002)$ & 0.031 \\
\hline AST (U/L) & $1.002(1.001-1.003)$ & $<0.001$ & $1.002(1.001-1.003)$ & $<0.001$ \\
\hline 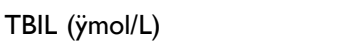 & $1.000(1.000-1.001)$ & 0.015 & $1.000(1.000-1.001)$ & 0.047 \\
\hline PLT $\left(\times 10^{9} / \mathrm{L}\right)$ & $1.002(1.000-1.004)$ & 0.020 & $1.002(1.000-1.004)$ & 0.043 \\
\hline AFP: $>200 / \leq 200 \mathrm{ng} / \mathrm{mL}$ & $2.095(1.527-2.874)$ & $<0.001$ & $2.391(1.743-3.280)$ & $<0.001$ \\
\hline Child-Pugh: A/B & $1.70 I(1.052-2.752)$ & 0.030 & $1.550(0.959-2.505)$ & 0.074 \\
\hline Ascites: yes/no & $1.358(0.846-2.180)$ & 0.205 & $1.450(0.904-2.323)$ & 0.123 \\
\hline Tumor size & $1.162(1.121-1.203)$ & $<0.001$ & $1.169(1.129-1.211)$ & $<0.001$ \\
\hline Tumor number: Multiple/single & $1.321(1.058-1.649)$ & 0.014 & $1.508(1.201-1.893)$ & $<0.001$ \\
\hline AIAG: $>0.91 / \leq 0.91$ & $1.754(1.266-2.434)$ & 0.001 & $1.693(1.222-2.346)$ & 0.002 \\
\hline Cirrhosis: yes/no & $0.730(0.531-1.003)$ & 0.052 & $0.705(0.515-0.966)$ & 0.029 \\
\hline BCLC:0-A/B & $2.848(2.020-4.012)$ & $<0.001$ & $3.217(2.283-4.534)$ & $<0.001$ \\
\hline
\end{tabular}

Abbreviations: OS, overall survival; RFS, recurrence-free survival; HR, hazard ratio; ALT, alanine aminotransferase; AST, aspartate aminotransferase; TBIL, total bilirubin; PLT, platelet; AFP, $\alpha$-fetoprotein; AIAG, age, international standardized ratio, albumin, $\gamma$-glutamyl transpeptidase; BCLC, Barcelona Clinic Liver Cancer. 
Table 3 Multivariate Analysis of Factors Associated with Overall Survival and Recurrence-Free Survival of Patients with Hepatitis B-Related Hepatocellular Carcinoma

\begin{tabular}{|c|c|c|c|c|}
\hline \multirow[t]{2}{*}{ Variables } & \multicolumn{2}{|c|}{ os } & \multicolumn{2}{|c|}{ RFS } \\
\hline & HR (95\% Cl) & $P$ & HR $(95 \% \mathrm{Cl})$ & $P$ \\
\hline ALT (U/L) & $0.998(0.995-1.001)$ & 0.285 & $0.998(0.995-I .00 I)$ & 0.289 \\
\hline AST (U/L) & $1.003(1.000-1.006)$ & 0.071 & $1.003(1.000-1.006)$ & 0.095 \\
\hline TBIL $(\mu \mathrm{mol} / \mathrm{L})$ & $1.000(1.000-1.001)$ & 0.128 & $1.000(1.000-1.001)$ & 0.346 \\
\hline PLT $\left(\times 10^{9} / \mathrm{l}\right)$ & $1.002(1.000-1.004)$ & 0.048 & $1.001(0.999-1.004)$ & 0.174 \\
\hline AFP:>200/ $\leq 200 \mathrm{ng} / \mathrm{mL}$ & $1.500(1.058-2.125)$ & 0.023 & $1.769(1.253-2.498)$ & 0.001 \\
\hline Child-Pugh: A/B & $0.97 \mid(0.557-I .695)$ & 0.918 & $1.000(0.580-1.722)$ & 0.999 \\
\hline Tumor Size & $1.107(1.049-1.168)$ & 0.000 & $1.105(1.047-1.165)$ & 0.000 \\
\hline Tumor number: Multiple/single & I.I75(0.934-I.477) & 0.168 & $1.355(1.069-1.719)$ & 0.012 \\
\hline AIAG & $3.47 I(I .302-9.25 I)$ & 0.013 & $2.726(1.012-7.344)$ & 0.047 \\
\hline$B C L C: 0-A / B$ & $1.246(0.764-2.034)$ & 0.378 & $1.364(0.839-2.215)$ & 0.210 \\
\hline
\end{tabular}

Abbreviations: OS, overall survival; RFS, recurrence-free survival; HR, hazard ratio; ALT, alanine aminotransferase; AST, aspartate aminotransferase; TBIL, total bilirubin; PLT, platelet; AFP, $\alpha$-fetoprotein; AIAG, age, international standardized ratio, albumin, $\gamma$-glutamyl transpeptidase; BCLC, Barcelona Clinic Liver Cancer.

Table 4 Ranking of AUC of the Prognostic Systems

\begin{tabular}{|l|l|l|l|}
\hline Rank & System & AUC & $\mathbf{9 5 \%} \mathbf{C l}$ \\
\hline 1 & AIAG+ tumor size+ AFP stage & 0.728 & $0.676-0.769$ \\
2 & AIAG+ tumor size & 0.723 & $0.676-0.769$ \\
3 & BCLC stage & 0.654 & $0.602-0.705$ \\
4 & AIAG+ AFP stage & 0.608 & $0.555-0.662$ \\
5 & AIAG & 0.574 & $0.521-0.628$ \\
\hline
\end{tabular}

Abbreviations: AUC, area under curve; AIAG, age, international standardized ratio, albumin, $\gamma$-glutamyl transpeptidase; AFP, $\alpha$-fetoprotein; BCLC Barcelona Clinic Liver Cancer.

including AFP, tumor size and tumor number, could affect the prognosis of HCC. Among the above mentioned indexes for evaluating liver function, Child-Pugh grade and BCLC stage, AIAG, tumor size, AFP and tumor number were risk factors for OS and RFS in our study.

In clinical practice, the BCLC staging was an important parameter for developing treatment strategy and evaluating the prognosis of HCC patients. ${ }^{23,24}$ More recently, the BCLC staging has also been used for further prognostication of HCC patients undergoing radiation therapy, radiofrequency ablation, ${ }^{25,26}$ transarterial chemoembolization, ${ }^{27}$ and even of HCC patients without treatment. ${ }^{28,29}$ However, BCLC staging mainly focuses on tumor characteristics that cannot be modified, and is not related to cirrhotic status. ${ }^{30}$ In addition, the data applied in BCLC were collected from several small Western cohorts of patients with predominant HCV infection. ${ }^{31}$ Therefore, this model may not be suitable for Asian countries, where the main cause of HCC is HBV infection. ${ }^{32}$ In our study, we initially assessed the prognostic significance of AIAG for HBV-related HCC. However, the predictive accuracy of AIAG alone did not perform very well, with 0.91 of the optimal cut-off value, $37.2 \%$ sensitivity, $75.7 \%$ specificity, and 0.574 of AUC value (95\% CI: 0.521-0.628, $p=0.008$ ), which might be caused by the small sample size and bias from data structures. To maximize the accuracy, two risk factors (tumor size and AFP) were selected to build the model. Our results suggested that the inclusion of tumor size and AFP could improve the reliability and clinical significance of the model. Furthermore, our newly established model showed that the predictive value of the

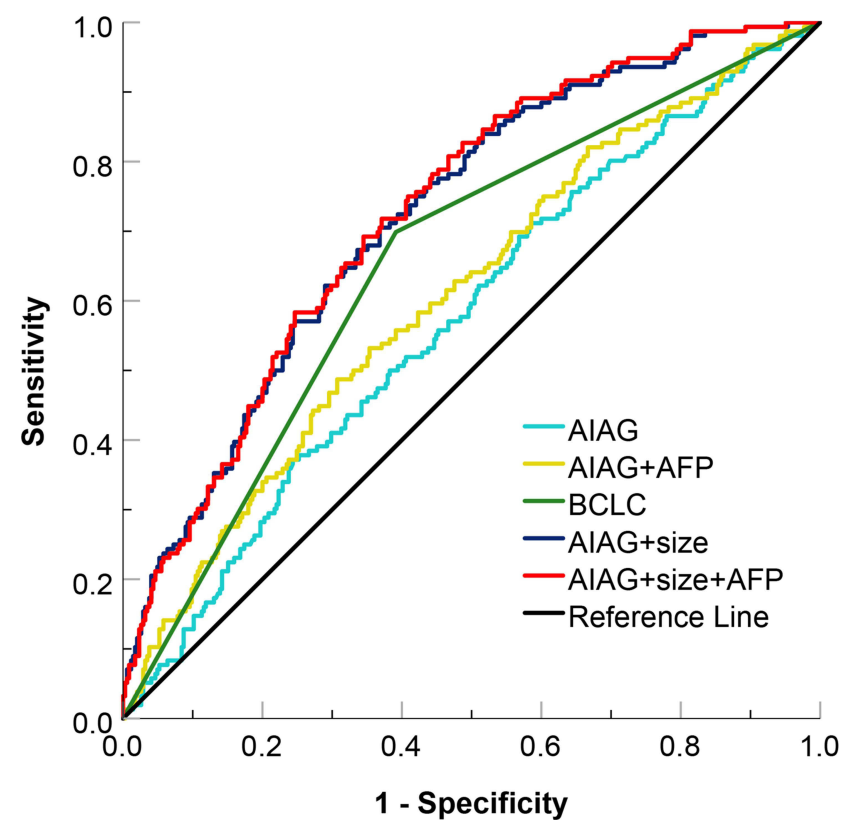

Figure 5 ROC curves of the subgroups stratified according to the AIAG, tumor size and AFP. 
Table 5 Ranking of Discriminatory Ability of the Prognostic Systems on the Basis of the Concordance Index

\begin{tabular}{|l|l|l|l|}
\hline Rank & System & C Index & $\mathbf{9 5 \%} \mathbf{C l}$ \\
\hline 1 & AIAG+ tumor size+ AFP stage & 0.710 & $0.669-0.75 I$ \\
2 & AIAG+ tumor size & 0.707 & $0.666-0.749$ \\
3 & BCLC stage & 0.636 & $0.598-0.673$ \\
4 & AIAG+ AFP stage & 0.611 & $0.564-0.658$ \\
5 & AlAG & 0.525 & $0.490-0.559$ \\
\hline
\end{tabular}

Abbreviations: $\mathrm{Cl}$, confidence interval; $\mathrm{AIAG}$, age, international standardized ratio, albumin, $\gamma$-glutamyl transpeptidase; AFP, $\alpha$-fetoprotein; BCLC, Barcelona Clinic Liver Cancer.

combination of AIAG, tumor size and AFP was better than the classic BCLC stage for the prognosis of HCC patients.

To control the potential confusion and bias, we exclusively recruited patients with HCC developing from HBV, and patients with other etiologies or without radical therapy were excluded. The four variables of AIAG were considered to be important prognostic factors of HCC. It has been reported that compared with many tumor factors, HCC patients' age was more relevant with the outcome. ${ }^{33}$ Kaibori et al found that the OS of patients aged greater than 75 years old was significantly lesser than that of patients aged less than 75 years old, and there was no significant difference in the disease-free survival among different age groups. ${ }^{34} \mathrm{~A}$ research identified age $\geq 70$ years old and liver cirrhosis as the independent prognostic factors of postoperative morbidity. ${ }^{33}$

A study including 2509 HCC patients showed that patients with low INR had significantly worse OS and RFS than those with the normal or high INR. ${ }^{35}$ In addition, it was found that HCC patients with a low INR level, which reflected on a hypercoagulability state, had a significantly higher incidence of MVI than patients with a normal or high INR level. Unstable hemostatic status of cirrhosis could easily be tipped towards the development of HCC; however, the detailed underlying mechanisms need to be further studied. Wang et al reported that ALB and GGT were independent prognostic factors of OS and RFS in HCC patients. ${ }^{36}$ This may be related to the fact that ALB itself could suppress human HCC proliferation and cell cycle. ${ }^{37}$ In addition, GGT, as a cell surface enzyme, can be used as a marker of many diseases. ${ }^{38}$ GGT can promote tumor progression and induce bad prognosis through damaging DNA and inducing genome instability, releasing reactive oxygen species to activate invasion-related signaling pathway, blocking chemotherapy, regulating microRNAs, and managing $\mathrm{CpG}$ island methylation. ${ }^{39}$
AFP and tumor size have been used in many prognostic models of HCC, which were demonstrated to have great prediction ability and evaluation effect. AFP is a widely used biomarker to monitor patients at risk of developing $\mathrm{HCC}$ and assess the treatment response of patients with HCC. Importantly, the high expression of AFP is a recognized manifestation of the aggressive biological behavior of HCC. Both AFP and tumor size can be used as a reflection of tumor burden. At the same time, AFP and tumor size were predictors of patient survival in our study, so we used them as elements for building a more comprehensive scoring model.

There are some limitations in our study. Firstly, the samples were only from two hospitals and it was a doublecenter retrospective study. Secondly, the quality of the retrospective study cannot be well controlled, so that it may be unavoidable to have problems such as confounding, bias, some missing values and non-standardization of results. Last but not least, preoperative use of antiinfection medication, hematological diseases, albumin infusion and blood transfusion may affect the evaluation model. Therefore, a multiple-center study with quality controlled well and complete data of patients should be done in the future to explore the predictive role of the combination of AIAG, tumor size and AFP stage.

\section{Conclusions}

In conclusion, AIAG is an effective indicator to predict OS and RFS for hepatitis B-related HCC patients who received radical treatment. AIAG is significantly associated with the survival rate of HCC patients, and provides additional prognostic information for patients with HCC. Our findings suggest that the combination of AIAG, tumor size and AFP stage has a better predictive value for the prognosis of patients with hepatitis B-related hepatocellular carcinoma. However, in the future, more further studies are necessary to investigate whether our findings would be applicable to HCC patients who received other therapies.

\section{Data Sharing Statement}

The raw datasets generated during the current study are available from Yi Tan upon reasonable request.

\section{Ethical Considerations}

This study was conducted in accordance with the declaration of Helsinki. This study was conducted with approval from the Ethics Committee of our hospitals. Written informed consent was obtained from the participants. 


\section{Author Contributions}

All authors made a significant contribution to the work reported, whether that is in the conception, study design, execution, acquisition of data, analysis and interpretation, or in all these areas; took part in drafting, revising or critically reviewing the article; gave final approval of the version to be published; have agreed on the journal to which the article has been submitted; and agree to be accountable for all aspects of the work.

\section{Funding}

Science and Technological Fund of Anhui Province for Outstanding Youth (Grant No. 2008085J37), the First Affiliated Hospital of Bengbu Medical College Science Funding for Distinguished Young Scholars (Grant No. 2019byyfyjq05), the key project of Anhui Provincial Education Department (Grant No. KJ2018ZD022), the National Nature Science Foundation of China (Grant No. 81600452 ) and the project was supported by the Graduate Research Innovation Program of Bengbu Medical College (Grant No. Byycx20085). We thank for the supports from these funding sources.

\section{Disclosure}

The authors report no conflicts of interest in this work.

\section{References}

1. Siegel RL, Miller KD, Jemal A. Cancer statistics, 2020. CA Cancer J Clin. 2020;70(1):7-30. doi:10.3322/caac.21590

2. Buonaguro L, Petrizzo A, Tagliamonte M, et al. Challenges in cancer vaccine development for hepatocellular carcinoma. J Hepatol. 2013;59(4):897-903. doi:10.1016/j.jhep.2013.05.031

3. Kabbach G, Assi HA, Bolotin G, et al. Hepatobiliary tumors: update on diagnosis and management. $J$ Clin Transl Hepatol. 2015;3:169-181.

4. Jafri W, Kamran M. Hepatocellular carcinoma in Asia: a challenging situation. Euroasian J Hepatogastroenterol. 2019;9(1):27-33. doi:10.5005/jp-journals-10018-1292

5. Geh D, Anstee QM, Reeves HL. NAFLD-associated HCC: progress and opportunities. J Hepatocell Carcinoma. 2021;8:223-239. doi:10.2147/JHC.S272213

6. Sheka AC, Adeyi O, Thompson J, et al. Nonalcoholic steatohepatitis: a review. JAMA. 2020;323(12):1175-1183. doi:10.1001/jama.20 20.2298

7. Dhar D, Baglieri J, Kisseleva T, Brenner DA. Mechanisms of liver fibrosis and its role in liver cancer. Exp Biol Med (Maywood). 2020;245(2):96-108. doi:10.1177/1535370219898141

8. Bray F, Ferlay J, Soerjomataram I, et al. Global cancer statistics 2018: GLOBOCAN estimates of incidence and mortality worldwide for 36 cancers in 185 countries. CA Cancer J Clin. 2018;68 (6):394-424. doi:10.3322/caac. 21492

9. European Association for the Study of the Liver. Electronic address eee, European Association for the Study of the L. EASL clinical practice guidelines: management of hepatocellular carcinoma. J Hepatol. 2018;69:182-236.
10. Sasaki K, Shindoh J, Margonis GA, et al. Effect of background liver cirrhosis on outcomes of hepatectomy for hepatocellular carcinoma. JAMA Surg. 2017;152(3):e165059. doi:10.1001/jamasurg.2016.5059

11. Liu Y, Cheng C, Zhou H, et al. Comparison of Modified Child-Pugh (MCP), Albumin-bilirubin (ALBI), and Child-Pugh (CP) score for predicting of survival in hepatocellular carcinoma patients treated with transcatheter arterial chemoembolization. Bull Cancer. 2021: S0007-S4551. PMID: 34247763. doi:10.1016/j.bulcan.2021.04.017

12. Forner A, Reig ME, de Lope CR, Bruix J. Current strategy for staging and treatment: the BCLC update and future prospects. Semin Liver Dis. 2010;30(1):61-74. PMID: 20175034. doi:10.1055/ s-0030-1247133

13. Dong M, Wu J, Yu X, et al. Validation and comparison of seventeen noninvasive models for evaluating liver fibrosis in Chinese hepatitis B patients. Liver Int. 2018;38(9):1562-1570. doi:10.1111/liv.13688

14. Ho SY, Wang LC, Hsu CY, et al. Metavir fibrosis stage in hepatitis C-related hepatocellular carcinoma and association with noninvasive liver reserve models. J Gastrointest Surg. 2020;24(8):1860-1862. doi:10.1007/s11605-020-04627-1

15. Marasco G, Colecchia A, Silva G, et al. Non-invasive tests for the prediction of primary hepatocellular carcinoma. World J Gastroenterol. 2020;26(24):3326-3343. doi:10.3748/wjg.v26. i24.3326

16. Wu SD, Ni YJ, Liu LL, et al. Establishment and validation of a simple noninvasive model to predict significant liver fibrosis in patients with chronic hepatitis B. Hepatol Int. 2012;6(1):360-368. doi:10.1007/s12072-011-9328-1

17. Kanda T, Goto T, Hirotsu Y, Moriyama M, Omata M. Molecular mechanisms driving progression of liver cirrhosis towards hepatocellular carcinoma in chronic hepatitis $\mathrm{B}$ and $\mathrm{C}$ infections: a review. Int J Mol Sci. 2019;20(6):1358. doi:10.3390/ijms20061358

18. Zakaria HM, Mohamed A, Omar H, et al. Alpha-fetoprotein level to total tumor volume as a predictor of hepatocellular carcinoma recurrence after resection. A retrospective cohort study. Ann Med Surg. 2020;54:109-113. doi:10.1016/j.amsu.2020.04.014

19. Zakaria HM, Macshut M, Gaballa NK, et al. Total tumor volume as a prognostic value for survival following liver resection in patients with hepatocellular carcinoma. Retrospective cohort study. Ann Med Surg. 2020;54:47-53. doi:10.1016/j.amsu.2020.04.001

20. Liang L, Wang MD, Zhang YM, et al. Association of postoperative biomarker response with recurrence and survival in patients with hepatocellular carcinoma and high alpha-fetoprotein expressions (>400 ng/mL). J Hepatocell Carcinoma. 2021;8:103-118. doi:10.2147/JHC.S289840

21. Liu H, Yang Y, Chen C, et al. Reclassification of tumor size for solitary HBV-related hepatocellular carcinoma by minimum $\mathrm{p}$ value method: a large retrospective study. World J Surg Oncol. 2020;18 (1):185. doi:10.1186/s12957-020-01963-z

22. Kim MN, Hwang SG, Kim BK, et al. Liver cirrhosis, not antiviral therapy, predicts clinical outcome in cohorts with heterogeneous hepatitis B viral status. Gut Liver. 2019;13(2):197-205. doi:10.5009/gnl18204

23. Adhoute X, Penaranda G, Bronowicki JP, et al. Usefulness of the HKLC vs. the BCLC staging system in a European HCC cohort. J Hepatol. 2015;62(2):492-493. doi:10.1016/j.jhep.2014.08.035

24. Zhang YF, Shi M, Lu LH, Wang L, Guo RP. Selecting an optimal staging system for intermediate-stage hepatocellular carcinoma: comparison of 9 currently used prognostic models. J Hepatocell Carcinoma. 2021;8:253-261. doi:10.2147/JHC.S305581

25. Moctezuma-Velazquez C, Montano-Loza AJ, Meza-Junco J, et al. Selective internal radiation therapy for hepatocellular carcinoma across the Barcelona clinic liver cancer stages. Dig Dis Sci. 2021;66(3):899-911. doi:10.1007/s10620-020-06245-y

26. Kim N, Cheng J, Jung I, et al. Stereotactic body radiation therapy vs. radiofrequency ablation in Asian patients with hepatocellular carcinoma. J Hepatol. 2020;73(1):121-129. doi:10.1016/j.jhep.2020.03.005 
27. Labgaa I, Taffe P, Martin D, et al. Comparison of partial hepatectomy and transarterial chemoembolization in intermediate-stage hepatocellular carcinoma: a systematic review and meta-analysis. Liver Cancer. 2020;9(2):138-147. doi:10.1159/000505093

28. Giannini EG, Farinati F, Ciccarese F, et al. Prognosis of untreated hepatocellular carcinoma. Hepatology. 2015;61(1):184-190. doi:10.1002/hep. 27443

29. Giannini EG, Moscatelli A, Pellegatta G, et al. Application of the intermediate-stage subclassification to patients with untreated hepatocellular carcinoma. Am J Gastroenterol. 2016;111(1):70-77. doi:10.1038/ajg.2015.389

30. Tellapuri S, Sutphin PD, Beg MS, et al. Staging systems of hepatocellular carcinoma: a review. Indian J Gastroenterol. 2018;37 (6):481-491. doi:10.1007/s12664-018-0915-0

31. Llovet JM, Bru C, Bruix J. Prognosis of hepatocellular carcinoma: the BCLC staging classification. Semin Liver Dis. 1999;19 (03):329-338. doi:10.1055/s-2007-1007122

32. Yu SJ. A concise review of updated guidelines regarding the management of hepatocellular carcinoma around the world: 2010-2016. Clin Mol Hepatol. 2016;22(1):7-17. doi:10.3350/cmh.2016.22.1.7

33. Galun D, Bogdanovic A, Zivanovic M, Zuvela M. Short- and long-term outcomes after hepatectomy in elderly patients with hepatocellular carcinoma: an analysis of 229 cases from a developing country. J Hepatocell Carcinoma. 2021;8:155-165

34. Kaibori M, Yoshii K, Yokota I, et al. Impact of advanced age on survival in patients undergoing resection of hepatocellular carcinoma: report of a Japanese nationwide survey. Ann Surg. 2019;269 (4):692-699. doi:10.1097/SLA.0000000000002526
35. Zhang XP, Zhou TF, Wang ZH, et al. Association of preoperative hypercoagulability with poor prognosis in hepatocellular carcinoma patients with microvascular invasion after liver resection: a multicenter study. Ann Surg Oncol. 2019;26(12):4117-4125. doi:10.1245/s10434-019-07504-7

36. Wang L, Li Q, Zhang J, et al. A novel prognostic scoring model based on albumin and gamma-glutamyltransferase for hepatocellular carcinoma prognosis. Cancer Manag Res. 2019;11:10685-10694. doi:10.2147/CMAR.S232073

37. Nojiri S, Joh T. Albumin suppresses human hepatocellular carcinoma proliferation and the cell cycle. Int J Mol Sci. 2014;15(3):5163-5174. doi:10.3390/ijms 15035163

38. Li S, Xu W, Liao M, et al. The significance of gamma-glutamyl transpeptidase to lymphocyte count ratio in the early postoperative recurrence monitoring and prognosis prediction of AFP-negative hepatocellular carcinoma. J Hepatocell Carcinoma. 2021;8:23-33. doi: $10.2147 /$ JHC.S286213

39. Xia J, Song P, Sun Z, et al. Advances of diagnostic and mechanistic studies of gamma-glutamyl transpeptidase in hepatocellular carcinoma. Drug Discov Ther. 2016;10(4):181-187. doi:10.5582/ ddt.2016.01052
International Journal of General Medicine

\section{Publish your work in this journal}

The International Journal of General Medicine is an international, peer-reviewed open-access journal that focuses on general and internal medicine, pathogenesis, epidemiology, diagnosis, monitoring and treatment protocols. The journal is characterized by the rapid reporting of reviews, original research and clinical studies

\section{Dovepress}

across all disease areas. The manuscript management system is completely online and includes a very quick and fair peer-review system, which is all easy to use. Visit http://www.dovepress.com/ testimonials.php to read real quotes from published authors. 